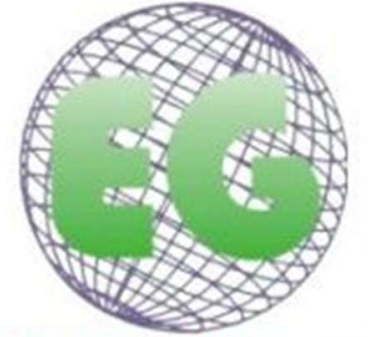

ISSN 1695-6141 $N^{\circ} 60$

\title{
Medición de la calidad de vida en adultos mayores
} institucionalizados de Lima (Perú)

Measuring the quality of life in institutionalized seniors in Lima (Peru)

\author{
Stephanie Antonella Queirolo Ore ${ }^{1}$ \\ Miguel Barboza Palomino ${ }^{2}$ \\ José Ventura-León ${ }^{2}$ \\ 1 Universidad Peruana de Ciencias Aplicadas. Lima. Perú. queirolostephanie@gmail.com
2 Universidad Privada del Norte. Perú.
}

\section{https://doi.org/10.6018/eglobal.420681}

Recibido: $30 / 03 / 2020$

Aceptado: $7 / 05 / 2020$

\section{RESUMEN:}

Introducción: Los adultos mayores experimentan cambios a nivel físico, cognitivo, social y emocional que tienen un impacto en su calidad de vida. Por lo tanto, resulta necesario disponer de instrumentos con adecuadas propiedades de medición para identificar y medir esta variable, de modo que las inferencias o interpretaciones sean válidas para la toma de decisiones.

Objetivo: El objetivo del estudio fue analizar las propiedades de medición del cuestionario de calidad de vida WHOQOL-OLD en adultos mayores peruanos institucionalizados.

Método: Participaron 300 adultos mayores de 65 años (Medad=78.41) institucionalizados en un asilo de la ciudad de Lima, donde 173 (57.7\%) fueron hombres y 127 (42.3\%) fueron mujeres. Se analizó la estructura interna del WHOQOL-OLD a través del análisis factorial confirmatorio (AFC) y la validez convergente con el Índice de Calidad de Vida (ICV). Asimismo, se estimaron puntuaciones de fiabilidad y se obtuvo un baremo para la muestra de estudio.

Resultados: Los resultados indican que la estructura de seis factores del WHOQOL-OLD presenta un ajuste satisfactorio con los datos $(\mathrm{CFI}=.97 ; \mathrm{TLI}=.97 ; \mathrm{SRMR}=.02 ; \mathrm{RMSEA}=.06)$ y una adecuada consistencia interna (alfa de Cronbach entre .94 y .98). Asimismo, se evidencian relaciones significativas con las dimensiones autonomía $\left(r_{s}=.13, p<.05\right)$ y participación social del ICV $\left(r_{s}=.16, p\right.$ $<.01)$.

Conclusiones: El cuestionario de calidad de vida WHOQOL-OLD cuenta con evidencias empíricas de validez y fiabilidad que lo sitúan como un instrumento adecuado para medir la variable de la calidad de vida.

Palabras clave: Adultos mayores, Calidad de vida, Cuestionario WHOQOL-OLD.

\section{ABSTRACT:}

Introduction: Older adults experience physical, cognitive, social, and emotional changes that impact their quality of life. It is therefore necessary to have instruments with appropriate measurement properties to identify and measure this variable, so that inferences or interpretations are valid for decision-making.

Objective: This study analyzes the measurement properties of the WHOQOL-OLD questionnaire for Peruvian institutionalized seniors. 
Method: Participants included 300 adults over 65 years $\left(M_{a g e}=78.41\right)$ institutionalized in a nursing home in the city of Lima, of which $173(57.7 \%)$ were men and $127(42.3 \%)$ were women. The internal structure of the WHOQOL-OLD was assessed through confirmatory factor analysis (CFA), whereas the convergent validity was analyzed using the Quality of Life Index (QLI). In addition, reliability scores were calculated and a scale for the study sample was obtained.

Results: The results show that the structure of the six factors involved in the WHOQOL-OLD is suitably adjusted to data $(\mathrm{CFI}=.97 ; \mathrm{TLI}=.97 ; \mathrm{SRMR}=.02$; RMSEA $=.06)$, with proper internal consistency (Cronbach's a ranging from .94 to .98). Likewise, it is possible to observe significant relationships with the autonomy $\left(r_{s}=.13, p<.05\right)$ and social involvement $\left(r_{s}=.16, p<.01\right)$ dimensions included in the QLI.

Conclusions: The WHOQOL-OLD Quality of Life Questionnaire has empirical evidence of validity and reliability that makes it an appropriate instrument to measure the quality of life variable.

Keywords: Seniors, Quality of life, WHOQOL-OLD questionnaire

\section{INTRODUCCIÓN}

En los últimos años, la población de adultos mayores (AM) en el mundo ha ido en aumento, debido al incremento de la esperanza de vida y a la disminución de la tasa de fecundidad ${ }^{(1)}$. Esta es una realidad que también se visualiza en el Perú, donde se estima que en el 2025 esta población represente el $12 \%$ del total ${ }^{(2)}$. Durante la tercera edad, los AM experimentan diferentes cambios a nivel físico, cognitivo, emocional y social que tienen un impacto en su calidad de vida ${ }^{(3,4)}$. Al respecto, la Organización Mundial de la Salud (OMS) ${ }^{(1)}$ define a la calidad de vida como la percepción de una persona sobre su existencia en el contexto de su cultura y del sistema de valores en el que vive y en relación con sus objetivos, sus expectativas, sus normas e inquietudes.

Existe evidencia que señala que la calidad de vida en los AM se asocia con variables vinculadas con la salud física y psicológica. De esta forma, por ejemplo, investigaciones previas indican que la salud percibida ${ }^{(5)}$ y el funcionamiento cognitivo(6) influyen en la percepción de la calidad de vida. Así también, existe una relación positiva entre la calidad de vida, el bienestar y la satisfacción con la vida $(7,8)$. Además, una buena percepción de la calidad de vida influye en el funcionamiento comportamental, la voluntad de vivir y el envejecimiento exitoso ${ }^{(6,9)}$.

Como se ha expuesto, la calidad de vida es un concepto multidimensional que se relaciona con distintas variables. En el caso de los AM se espera que esta sea construida de forma favorable, de modo que reconozcan y vivan la vejez como una etapa de continuación de un proceso vital y no como una fase de declinación funcional y aislamiento social (10). En la construcción de una percepción adecuada de la calidad de vida también influyen los vínculos positivos y factores como el contexto familiar y social ${ }^{(4,11)}$. No obstante, en muchas situaciones las familias no pueden hacerse responsables de los AM, encontrándose a muchos de estos en los asilos ${ }^{(12)}$.

Considerando la importancia de la calidad de vida y su relación con la salud física y psicológica de los $A M{ }^{(5,9)}$, resulta necesario disponer de instrumentos para poder medirla. Estos deben evidenciar adecuadas propiedades de medición, de modo que se garantiza que las inferencias o interpretaciones que se realicen a partir de las puntuaciones sean válidas, pudiendo tomarse decisiones acertadas para desarrollar actividades de prevención e intervención individual o grupal. 
A la fecha, se ha incrementado el interés por medir la calidad de vida y se disponen de algunos instrumentos en el ámbito de la salud (13). Es así como la OMS ha desarrollado el World Health Organization Quality of Life-Old (WHOQOL-OLD) con la finalidad de conocer la situación de la calidad de vida de los AM a nivel mundial. El instrumento, en su versión original, presenta una estructura de seis dimensiones con 24 ítems y una puntuación de fiabilidad de $.89{ }^{(14)}$. Este instrumento recientemente se ha adaptado a la realidad de algunos países latinoamericanos como México, Ecuador y Chile.

Una adaptación realizada en México demostró una estructura interna de seis dimensiones y se reporta una fiabilidad de alfa de Cronbach ( $\alpha$ ) de .84 (15). Otro estudio, también desarrollado en México, da cuenta de una estructura de cuatro dimensiones eliminando cuatro ítems y una puntuación de fiabilidad de a de .89 (16). Por su parte, en Chile, se verifica la estructura interna de seis dimensiones y se reporta un $\alpha$ de $.80{ }^{(17)}$. Así también, en el Ecuador, se comunica una estructura interna de cuatro dimensiones y un a de $.84^{(18)}$.

A pesar de estos esfuerzos, aún resulta necesario continuar desarrollando estudios de adaptación de este instrumento a otros países, tal es el caso del Perú. Por lo tanto, el objetivo de la presente investigación es analizar las propiedades de medición del cuestionario de calidad de vida WHOQOL-OLD en una muestra de adultos mayores institucionalizados de Lima.

\section{MÉTODO}

Esta investigación corresponde a un diseño instrumental, puesto que se analizaron las propiedades de medición del WHOQOL-OLD ${ }^{(19)}$.

\section{Muestra}

La población de estudio fueron 536 AM institucionalizados en un asilo de la ciudad de Lima. De este total, se seleccionó una muestra de 300 AM de 65 años o más (M edad =78.41), que cumplieron con los criterios de inclusión de no presentar un diagnóstico previo de algún deterioro cognitivo (moderado o severo) o manifestaciones depresivas. Los participantes fueron identificados y seleccionados considerando una base de datos proporcionada por la institución y elaborada a partir de una evaluación continua de tamizaje cognitivo y afectivo.

Del total de la muestra, $127(42,3 \%)$ fueron hombres y $173(57,7 \%)$ fueron mujeres, siendo la edad mínima 65 y la máxima 96. En cuanto corresponde a su grado instrucción, el $24.3 \%$ tiene primaria completa, $17.7 \%$ primaria incompleta, $33.7 \%$ secundaria completa, $6.7 \%$ secundaria incompleta $13.7 \%$ superior completa y $4 \%$ superior incompleta. Por su parte, en lo que respecta a su estado civil, el $14.3 \%$ son casados, $13 \%$ divorciados, $57.3 \%$ solteros, $15.3 \%$ viudos.

Para la selección de los participantes se realizó un muestreo de tipo no probabilístico intencional y siguiendo los criterios de inclusión se identificó una muestra de 300 AM. Considerando que en el estudio se empleó la técnica del análisis factorial, que suele recomendar que por cada ítem administrado se cuente con al menos 5 o 10 personas, el número de participantes seleccionados es el adecuado y cumple con esta condición ${ }^{(20)}$. 


\section{Instrumentos}

\section{Escala de calidad de vida WHOQOL-OLD}

Para la medición de la calidad de vida se aplicó la escala de calidad de vida en adultos mayores World Health Organization Quality of Life of Older Adults WHOQOLOLD de la OMS. Para fines de la presente investigación se hizo uso del cuestionario adaptado en México ${ }^{(15)}$. Esta escala consta de 24 ítems de tipo Likert organizados en 6 dimensiones de 4 ítems: 1) Habilidades sensoriales (Hs), 2) Autonomía (Aut), 3) Actividades pasadas, presentes y futuras (Appf), 4) Participación social (Ps), 5) Muerte (Ma), 6) Intimidad (Int). Los puntajes de cada dimensión pueden variar entre 4 y 20 puntos ${ }^{(14)}$.

\section{Índice de Calidad de Vida (ICV)}

EI ICV mide aspectos de la calidad de vida: bienestar psicológico, bienestar físico, autocuidado y funcionamiento independiente, funcionamiento ocupacional, funcionamiento interpersonal, apoyo emocional y social, apoyo comunitario y de servicios, plenitud personal, satisfacción espiritual y una valoración global de la calidad de vida. Cada uno de estos aspectos se representa en diez ítems, los cuales son valorados en una escala ordinal de 1 a 10 puntos. Este instrumento se encuentra validado para la población peruana y se reporta un $\alpha$ de $.87^{(21)}$.

\section{Procedimiento}

El protocolo de la investigación fue aprobado por el Comité de Ética de la Universidad Peruana de Ciencias Aplicadas. Luego de la aprobación, se solicitó el permiso de uso de la prueba a los autores de la adaptación realizada en México ${ }^{(15)}$. A su vez, se realizó una revisión bibliográfica de los instrumentos utilizados para medir la calidad de vida en los adultos mayores y se seleccionó un instrumento para fines de analizar la validez convergente. A continuación, se efectuaron las coordinaciones de presentación del proyecto y solicitud de permiso para la recolección de datos en un asilo de Lima. Después de haber obtenido la autorización, se procedió a la aplicación de ambos instrumentos. En esta fase de la investigación, se hizo entrega previa de un consentimiento informado a los participantes del estudio.

Inicialmente, en el proceso de la recolección de datos se realizó una prueba piloto con 27 AM. El propósito de esta actividad fue identificar si existían dificultades en la comprensión de los ítems o en las instrucciones de cada prueba. Los instrumentos fueron aplicados de forma individual e implicó la lectura de cada pregunta y de las alternativas de respuesta, modulándose el tono de voz según las características de cada participante.

Para poder analizar los datos de la prueba se hizo uso del programa IBM SPSS v. 25. En primer lugar, se realizaron los análisis descriptivos y de homogeneidad con la finalidad de conocer la distribución de las respuestas; además de evidenciar la correlación total de los elementos. Después, se ejecutó el análisis factorial confirmatorio de manera que se compararon los modelos y sus índices de bondad de ajuste, lo cual permitió conocer la estructura interna. Luego, se procedió a realizar el análisis de las puntuaciones de fiabilidad, calculándose los coeficientes de alfa y Omega. Se utilizó el coeficiente Alfa para comparar la puntuación de fiabilidad con lo 
reportado en estudios previos y el coeficiente Omega por ser sugerido para el desarrollo de estudios factoriales ${ }^{(22)}$.

El proyecto de investigación fue aprobado en marzo de 2019 y ejecutado a partir de mayo del mismo año. En los meses de mayo y junio de 2019 se realizó la recolección de información. Después, de julio a diciembre de 2019 se elaboró la base de datos y se desarrollaron los procesos de análisis. Finalmente, entre enero y marzo de 2020 se redactó el informe final de investigación.

\section{RESULTADOS}

\section{Análisis descriptivos y de homogeneidad de ítems}

A partir del análisis descriptivo de los ítems, se observa una tendencia de respuestas de valores medios ya que las medias de los ítems han estado entre 2.43 y 4.04 , teniendo como referencias que los valores de respuesta de la escala van del 1 al 5 . Al analizar la asimetría y la curtosis se observa que los ítems se aproximan a una distribución normal y no hay excesos de asimetría, ya que ningún ítem supera el $1.5^{(23)}$. Se ha podido observar que en su mayoría los ítems muestran un adecuado grado de homogeneidad, pues cumplen con los criterios de presentar correlaciones ítem-test corregidas $\left(r_{\text {itc }}\right)$ mayores a .20 y son positivos ${ }^{(20)}$; sin embargo, el ítem 20 no cumple con los criterios de homogeneidad, motivo por el cual fue eliminado.

Tabla 1. Estadísticos descriptivos y correlaciones ítem-test corregidas del WHOQOL-OLD

\begin{tabular}{cccccc}
\hline Ítem & $\boldsymbol{M}$ & $\boldsymbol{D E}$ & $\boldsymbol{g 1}$ & $\boldsymbol{g} \mathbf{2}$ & $\boldsymbol{r}_{\text {itc }}$ \\
\hline 1 & 3.62 & 0.94 & 0.082 & -0.99 & 0.81 \\
2 & 3.59 & 1.26 & -0.147 & -1.4 & 0.82 \\
3 & 3.01 & 1 & 0.327 & -0.07 & 0.89 \\
4 & 2.6 & 1.03 & -0.197 & -0.58 & 0.88 \\
5 & 2.79 & 0.8 & -0.004 & -0.73 & 0.87 \\
6 & 3.65 & 0.97 & -0.195 & -0.18 & 0.86 \\
7 & 3.9 & 0.94 & -0.138 & -1.27 & 0.91 \\
8 & 4.04 & 0.91 & -0.491 & -0.8 & 0.9 \\
9 & 3.92 & 1.09 & -0.509 & -1.11 & 0.87 \\
10 & 3.82 & 1.01 & -0.273 & -1.1 & 0.79 \\
11 & 2.82 & 0.9 & -0.067 & -0.28 & 0.92 \\
12 & 2.92 & 1.09 & -0.046 & -0.7 & 0.87 \\
13 & 3.27 & 1.07 & -0.309 & -0.51 & 0.86 \\
14 & 2.64 & 1 & 0.171 & -0.47 & 0.83 \\
15 & 3.72 & 0.78 & -0.643 & 0.21 & 0.82 \\
16 & 3.35 & 0.96 & -0.698 & -0.24 & 0.93 \\
17 & 3.29 & 0.96 & -0.594 & -0.83 & 0.9 \\
18 & 3.45 & 0.95 & -0.463 & -0.35 & 0.91 \\
19 & 3.52 & 0.84 & -0.393 & -0.54 & 0.9 \\
20 & 3.27 & 0.84 & -0.272 & 0.74 & -0.65
\end{tabular}




$\begin{array}{llllll}21 & 2.72 & 1.05 & 0.045 & -0.37 & 0.9 \\ 22 & 2.66 & 1.12 & 0.279 & -0.39 & 0.94 \\ 23 & 2.62 & 1.26 & 0.255 & -1.02 & 0.93 \\ 24 & 2.43 & 1.29 & 0.493 & -0.87 & 0.93\end{array}$

Nota: $\mathrm{M}$ = media; $\mathrm{DE}$ = desviación estándar; g1= asimetría; g2 = curtosis; $\mathrm{r}_{\mathrm{itc}}=$ Correlación ítem test

\section{Análisis de validez basada en la estructura interna}

El modelo de seis factores del WHOQOL-OLD, retirando el ítem 20, presenta un

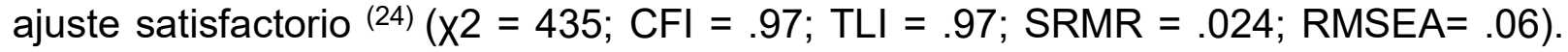
En la Tabla 2, se presenta la matriz de cargas factoriales de la escala sin el ítem 20. La dimensión de habilidades sociales presenta cargas factoriales con valores entre .92 y .95, la dimensión autonomía muestra cargas factoriales que van de .90 a .96, la dimensión de actividades presentes, pasadas y futuras tiene cargas factoriales entre .85 a .94, la dimensión de participación social cuenta con cargas factoriales que oscilan entre .84 y .97 , la dimensión de muerte y agonía presenta cargas factoriales de .89 a .94 y la dimensión de intimidad presenta valores de cargas factorial que van de .92 a .96. Por lo tanto, se confirma que todas las dimensiones cuentan con ítems con adecuadas cargas factoriales ya que superan el valor de $.30{ }^{(20)}$.

Tabla 2. Matriz factorial con cargas estandarizadas de WHOQOL-OLD

\begin{tabular}{|c|c|c|c|c|c|c|}
\hline \multirow{2}{*}{ Ítem } & \multicolumn{6}{|c|}{ Factor } \\
\hline & $\mathrm{Hs}$ & Aut & Appf & Ps & $\mathrm{Ma}$ & Int \\
\hline 1 & .92 & & & & & \\
\hline 2 & .95 & & & & & \\
\hline 10 & .92 & & & & & \\
\hline 3 & & .92 & & & & \\
\hline 4 & & .90 & & & & \\
\hline 5 & & .90 & & & & \\
\hline 11 & & .96 & & & & \\
\hline 12 & & & .91 & & & \\
\hline 13 & & & .90 & & & \\
\hline 15 & & & .85 & & & \\
\hline 19 & & & .94 & & & \\
\hline 14 & & & & .84 & & \\
\hline 16 & & & & .97 & & \\
\hline 17 & & & & .94 & & \\
\hline 18 & & & & .93 & & \\
\hline 6 & & & & & .89 & \\
\hline 7 & & & & & .94 & \\
\hline 8 & & & & & .93 & \\
\hline 9 & & & & & .90 & \\
\hline 21 & & & & & & .92 \\
\hline
\end{tabular}


Nota: $\overline{\mathrm{Hs}}=$ Habilidades sensoriales, Aut= Autonomía, Appf= Actividades presentes, pasadas y futuras, Ps=Participación social, Ma= Muerte y agonía, Int= Intimidad

\section{Evidencia de validez basada en la relación con otras variables}

Mediante la prueba de Kolmogorov-Smirnov se realizó un análisis de normalidad de los puntajes de cada una de las dimensiones del WHOQOL-OLD y del ICV. Se obtuvieron puntajes menores a 0.5 , por lo que se concluye que existe una distribución normal y se puede proceder a realizar el análisis de la validez convergente. Para este análisis se empleó el coeficiente de correlación de Spearman (rho).

En cuanto a la validez convergente, las dimensiones autonomía $\left(r_{s}=.13, p<.05\right)$ y participación social $\left(r_{s}=.16, p<.01\right)$ del WHOQOL-OLD tienen una relación positiva y significativa con el ICV. En las dimensiones restantes no se ha demostrado una relación significativa. En lo que corresponde a las relaciones intra dimensiones se ha evidenciado que, en su mayoría, salvo dos, no se demuestran relaciones significativas (ver Tabla 3).

Tabla 3. Coeficiente de correlación entre las dimensiones del WHOQOL-OLD e ICV

\begin{tabular}{cccccccc}
\hline Variables & 1 & 2 & 3 & 4 & 5 & 6 & 7 \\
\hline 1. Hs & 1 & & & & & & \\
2. Aut & -0.06 & 1 & & & & & \\
3. Appf & 0.02 & -0.01 & 1 & & & & \\
4. Ps & 0.05 & -0.05 & $.13^{*}$ & 1 & & & \\
5. Ma & -0.01 & -0.11 & -0.07 & -0.07 & 1 & & \\
6. Int & 0.05 & $.13^{*}$ & 0.03 & -0.02 & 0.07 & 1 & \\
7. ICV & 0.05 & $.13^{*}$ & 0.1 & $.16^{* *}$ & -0.03 & 0.04 & 1 \\
\hline
\end{tabular}

Nota: $\mathrm{Hs}=$ Habilidades sensoriales, Aut $=$ Autonomía, Appf $=$ Actividades presentes, pasadas $y$ futuras, Ps=Participación social, Ma= Muerte y agonía, Int= Intimidad.

${ }^{*} \mathrm{p}<.05,{ }^{* *} \mathrm{p}<.01$

\section{Análisis de fiabilidad por consistencia interna}

Se estimaron los coeficientes de alfa de Cronbach $(\alpha)$ y omega $(\omega)$. De esta forma, se obtuvieron los siguientes valores por cada dimensión: Habilidades sensoriales $(\alpha=$ $.94 ; \omega=.96)$, autonomía $(\alpha=.95 ; \omega=.96)$, actividades presentes, pasadas y futuras $(\alpha=.94 ; \omega=.94)$, participación social $(\alpha=.96 ; \omega=.96)$, muerte y agonía $(\alpha=.95 ; \omega=$ $.95)$, intimidad ( $\alpha=.97 ; \omega=.97$ ). Como se observa, todos los valores superan el .70 por lo que se demuestra una muy alta fiabilidad (25). 


\section{DISCUSIÓN}

La presente investigación tuvo como objetivo analizar las propiedades de medición de la Escala de Calidad de Vida WHOQOL-OLD en AM institucionalizados en un asilo de Lima (Perú). Los resultados evidencian que el instrumento presenta una estructura de 6 factores y validez convergente con otra medida de calidad de vida. Así también, cuenta con una alta fiabilidad. De esta forma, se dispone de un instrumento para la medición de la calidad de vida en AM peruanos.

Los resultados obtenidos se asemejan a los reportados por Acosta et al. ${ }^{(15)}$, al exhibir una estructura interna similar y una fiabilidad muy alta. En cuanto a la relación con otras variables, en el estudio citado se evidenció una validez divergente con las puntuaciones de una escala de depresión y una validez convergente con las puntuaciones de una escala de bienestar subjetivo, reportándose altas correlaciones. Esto no pudo evidenciarse en el presente estudio, donde se encontraron correlaciones parciales entre las dimensiones del WHOQOL-OLD y el ICV. Por lo tanto, se sugiere, en próximas investigaciones, incluir medidas de depresion y bienestar subjetivo en el proceso de validación del WHOQOL-OLD.

Por otra parte, Hernández et al. ${ }^{(16)}$ y Ortega et al. ${ }^{(18)}$ reportan estructuras internas que difieren de la estructura original y de lo encontrado en esta investigación. Sin embargo, se coincide en el hallazgo de una alta fiabilidad. A su vez, Urzúa y Navarrete ${ }^{(17)}$ comunican una estructura interna unidimensional con 6 ítems y una fiabilidad moderada. Esto debido a que desarrollaron un análisis de una versión abreviada del WHOQOL-OLD. Si bien, una versión corta puede facilitar el tiempo de aplicación de la prueba, se requiere en futuros estudios analizar la validez y fiabilidad de esta posible versión abreviada.

La presente investigación no está exenta de limitaciones, entre estas: a) la generalización de los resultados, b) el instrumento utilizado en la validez convergente. En lo que respecta a la generalización, los resultados de esta investigación no son concluyentes, debido a que el muestreo fue de tipo no probabilistico. Por lo tanto, en investigaciones posteriores se sugiere emplear muestreos de tipo probabilísticos.

Con relación al instrumento utilizado en la validez convergente, se hizo uso del ICV, el cual no contaba con la misma cantidad de dimesiones que el WHOQOL-OLD. Esto dificultó el procedimiento de análisis y encontrar posibles correlaciones significativas entre ambos instrumentos. Se sugiere que para próximas aplicaciones se tome en cuenta la estructura interna de los instrumentos que se utilicen para analizar la evidencia de validez convergente o divergente. Con base en lo encontrado por Acosta et al. ${ }^{(15)}$, se podría optar por analizar la convergencia del WHOQOL-OLD con medidas de depresión y bienestar subjetivo.

Al disponer de un instrumento que se evidencia tiene la capacidad de medir la calidad de vida de adultos mayores institucionalizados, este podría ser utilizado en actividades profesionales y de investigación. En el ámbito profesional, su uso puede ayudar a identificar a los adultos de acuerdo con su nivel de calidad de vida, facilitando la toma de decisiones para desarrollar intervenciones individuales y grupales. Por su parte, en el ámbito de la investigación, el instrumento puede emplearse para evaluar la efectividad de programas de intervención que trabajen la 
variable de la calidad de vida, así como, en el desarrollo de otro tipo de estudios que tomen en cuenta esta variable.

\section{CONCLUSIONES}

Se concluye que el WHOQOL-OLD mide la calidad de vida en adultos mayores institucionalizados de un asilo de la ciudad de Lima. De esta forma, se dispone de un instrumento cuyas inferencias e interpretaciones que se realicen a partir de sus puntuaciones son válidas y pueden ayudar a tomar decisiones en el ámbito de la salud en actividades de promoción o intervención individual o grupal vinculadas con la calidad de vida.

\section{REFERENCIAS}

1. Organización Mundial de la Salud. Envejecimiento activo: un marco político. Rev Esp Geriatr Gerontol. 2002; 37(S2): 74-105. Disponible en: http://envejecimiento.csic.es/documentos/documentos/oms-envejecimiento-01.pdf

2. Instituto Nacional de Estadística e Informática. Situación de la Población Adulta Mayor. Informe Técnico $\mathrm{N}^{\circ}$ 02. 2018. Disponible en: https://www.inei.gob.pe/media/MenuRecursivo/boletines/01-informe-tecnicon02 adulto ene-feb mar2018.pdf

3. Gimeno M, Macarro M, Muñoz L. El adulto: Etapas y consideraciones para el aprendizaje. Eúphoros. 2001. Disponible en: http://dialnet.unirioja.es/descarga/articulo/1183063.pdf

4. Molina Sena C, Mélendez Moral JC, Navarro Pardo E. Bienestar y calidad de vida en ancianos institucionalizados y no institucionalizados. An. Psicol. 2008; 24(2): 312-319. doi: 10.6018/analesps

5. Hernandez-Huayta J, Chavez-Meneses S, Carreazo NY. Salud y calidad de vida en adultos mayores de un área rural y urbana del Perú. Rev Peru Med Exp Salud Publica. 2016; 33(4): 680-688. doi: 10.17843/rpmesp.2016.334.2551

6. Caycho-Rodríguez T, Domínguez-Vergara J, Barboza-Palomino M. Funcionamiento cognitivo y comportamental, y calidad de vida en adultos mayores. Enferm Clin. 2019; 29(1): 57-58. doi:10.1016/j.enfcli.2018.04.001

7. Caycho-Rodríguez T, Ventura-León J, García-Cadena $\mathrm{CH}$, Tomás JM, Domínguez-Vergara J, Daniel L, Arias-Gallegos WL. Evidencias psicométricas de una medida breve de resiliencia en adultos mayores peruanos no institucionalizados. Psychosocial Intervention. 2018; 27(2): 73-79. doi: 10.5093/pi2018a6

8. Caycho-Rodríguez $\mathrm{T}$, Reyes Bossio M, Ventura-León J, Arias Gallegos WL, Domínguez-Vergara J, Azabache-Alvarado K. Evidencias psicométricas de una versión breve de la Coping Humor Scale en adultos mayores peruanos. Rev Esp Geriatr Gerontol. 2019; 54(4): 230-236. doi: 10.1016/j.regg.2018.09.012

9. Caycho-Rodríguez T, Rondón-Jara E, Domínguez-Vergara J, Cabrera-Orosco I. Voluntad de vivir en adultos mayores peruanos. Rev Esp Geriatr Gerontol. 2020; 55(2): 126-127. doi: 10.1016/j.regg.2019.09.006

10. Gutierrez R, Torres B. Calidad de vida y actitudes hacia la muerte en los adultos mayores de la microred 15 de Agosto de Paucarpata, Arequipa. 2016. Disponible en: http://repositorio.unsa.edu.pe/handle/UNSA/3520

11. Vera M. Significado de la calidad de vida del adulto mayor para sí mismo y para su familia. An. Facultad Med. 2013; 68(3): $284 . \quad$ doi: https://doi.org/10.15381/anales.v68i3.1218 
12. Méndez V. Cambios en el adulto mayor: percepciones sobre autonomía, bienestar y calidad de vida, en el hogar y/o residencias geriátricas. 2018. Disponible en: https://bit.ly/2QS5tKm

13. Gómez L, Verdugo M, Arias B, Navas P. Evaluación de la calidad de vida en personas mayores y con discapacidad: la Escala Fumat. Psychosocial Intervention. $\quad 2008 ; 17(2)$ : 189-199. Disponible en: http://scielo.isciii.es/pdf/inter/v17n2/v17n2a07.pdf

14. World Health Organization. Manual WHOQOL-OLD. European office Copenhagen. 2006. Disponible en: https://www.who.int/mental health/evidence/WHOQOL OLD Manual.pdf?ua=1

15. Acosta C, Echevarria S, Garcia R, Serrano D, Vales J. Confiabilidad y validez del Cuestionario de Calidad de Vida (WHOQOL-OLD) en adulto mayores mexicanos. Rev. Psicol. Salud. 2013; 23(2): 242-250. Disponible en: https://bit.ly/2wJwQ2A

16. Hernández J, Guadarrama R, Castillo S, Hernández G, Márquez O. Validación del WHOQOL-OLD en adultos mayores de México. PSIENCIA. Rev. Latinoam. Cienc. Psicol. 2015; 7(3): 397-405. doi: 10.5872/psiencia/7.3.21

17. Urzúa A, Navarret M. Calidad de vida en adultos mayores: Análisis factoriales de las versiones abreviadas del WHOQoL-Old en población Chilena. Rev. Med. Chil. 2013; 141(1): 28-33. doi: 10.4067/S0034-98872013000100004

18. Ortega A, Quinche A, Moreno E, Alvarez L. Validación del test WHOQOL-OLD para determinar la calidad de vida en los adultos mayores de centros geriátricos de la Ciudad de Loja - Ecuador. Ocronos Rev. Med. Enferm. 2018. Disponible en: https://revistamedica.com/validacion-test-whogol-old-calidad-de-vida/

19. Ato M, Lopez J, Benavente A. Un sistema de clasificación de los diseños de investigación en psicología. An. Psicol. 2013; 29(3): 1038-1059. doi: 10.6018/analesps.29.3.178511

20. Ferrando $P$, Anguiano $C$. El análisis factorial como técnica de investigación psicológica. Pap. Psicólogo. 2010; 31(1): 18-33. Disponible en: http://www.redalyc.org/pdf/778/77812441003.pdf

21. Robles Y, Saavedra J, Mezzich J, Sanez Y, Padilla M, Mejia O. Índice de calidad de vida: Validación en una muestra peruana. An. Salud Ment. 2016; 26(2): 33-46. Disponible

en: http://www.insm.gob.pe/ojsinsm/index.php/Revista1/article/view/75/56

22. Ventura-León JL, Caycho-Rodríguez T. El coeficiente Omega: un método alternativo para la estimación de la confiabilidad. Rev. Latinoam. Cienc. Soc. Niñez Juventud. 2017; 15(1): 625-627. Disponible en https://www.redalyc.org/jatsRepo/773/77349627039/html/index.html

23. Lloret Segura S, FerreresTraver A, Hernández Baeza A, Tomás Marco I. El análisis factorial exploratorio de los ítems: una guía práctica, revisada y actualizada. An. Psicol. 2014; 30(3): 1151-1169. doi: 10.6018/analesps.30.3.199361

24. Ruiz M, Pardo A, Martín R. Modelos de ecuaciones estructurales. Pap. Psicologo. 2010; 31(1): 34-45. Disponible en: http://www.papelesdelpsicologo.es/pdf/1794.pdf

25. Viladrich C, Angulo A, Doval E. Un viaje alrededor de alfa y omega para estimar la fiabilidad de consistencia interna. An. Psicol. 2017;33(3): 755-782. doi: https://doi.org/10.6018/analesps.33.3.268401 


\section{Apéndice A}

\section{CUESTIONARIO WHOQOL-OLD}

Instrucciones: Las siguientes preguntas hacen referencia a cuanto ha experimentado ciertos hechos en las dos últimas semanas. Por favor, responda las preguntas pensando en su vida en las dos ultimas semanas. Marque con una " $X$ " en la respuesta que mejor considere para cada pregunta.

1. ¿En qué medida los problemas con su vista, oído, gusto, olfato y tacto afectan su vida diaria?

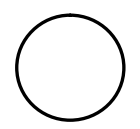

Nada

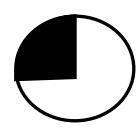

Un poco

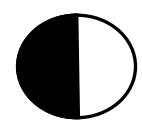

Lo normal

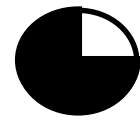

Bastante

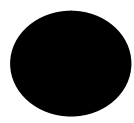

Extremadamente

2. ¿En qué medida la pérdida de su vista, oído, gusto, olfato y del tacto afecta su capacidad para participar en actividades?

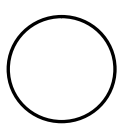

Nada

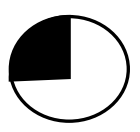

Un poco

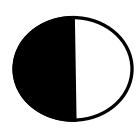

Lo norma

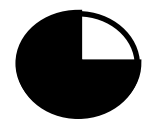

Bastante

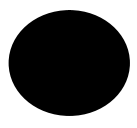

Extremadamente

3. ¿Cuánta libertad tiene para tomar sus propias decisiones?

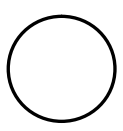

Nada

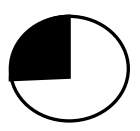

Un poco

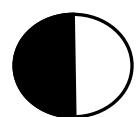

Lo normal

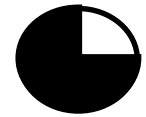

Bastante

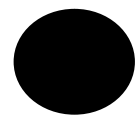

Extremadamente

4. ¿En qué medida siente que tiene control sobre su futuro?

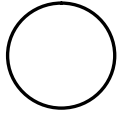

Nada

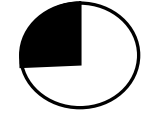

Un poco

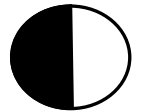

Lo normal

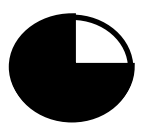

Bastante

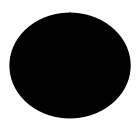

Extremadamente

5. ¿En qué grado siente que la gente que le rodea es respetuosa con su libertad?

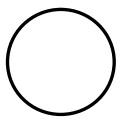

Nada

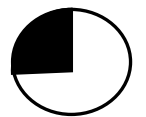

Un poco

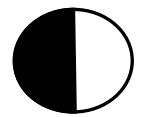

Lo normal

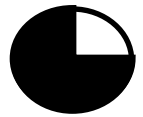

Bastante

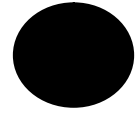

Extremadamente 
6. ¿En qué grado está preocupado(a) sobre la manera en que morirá?

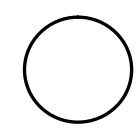

Nada

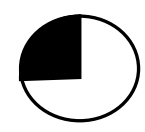

Un poco

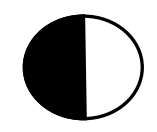

Lo normal

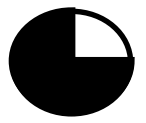

Bastante

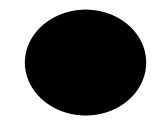

Extremadamente

7. ¿Cuánto miedo tiene de no ser capaz de controlar su muerte?

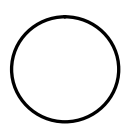

Nada

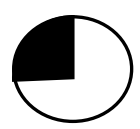

Un poco

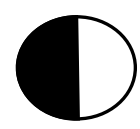

Lo normal

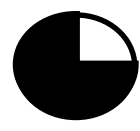

Bastante

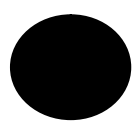

Extremadamente

8. ¿En qué grado está asustado(a) de morir?

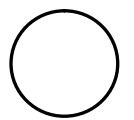

Nada

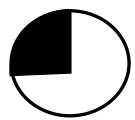

Un poco

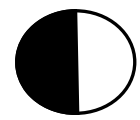

Lo norma

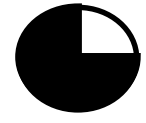

Bastante

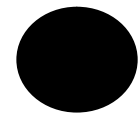

Extremadamente

9. ¿Cuánto teme tener dolor antes de morir?

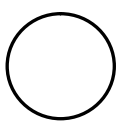

Nada

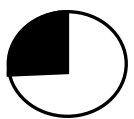

Un poco

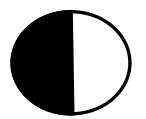

Lo normal

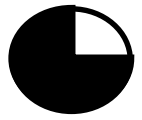

Bastante

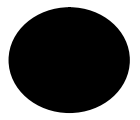

Extremadamente

Las siguientes preguntas hacen referencia en qué medida experimenta o fue capaz de hacer ciertas cosas en las últimas dos semanas, por ejemplo, haber salido tanto como quiso.

10. ¿En qué medida los problemas con su vista, oído, gusto, olfato y tacto, afectan su capacidad para relacionarse con los demás?

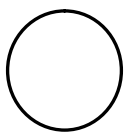

Nada

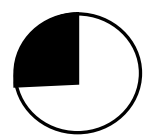

Un poco

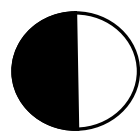

Moderadamente

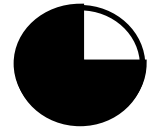

Bastante

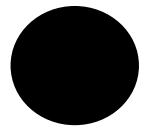

Totalmente

11. ¿En qué medida es capaz de hacer las cosas que le gustaría hacer?

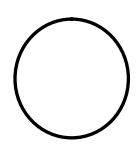

Nada

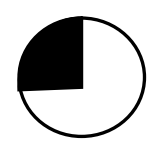

Un poco

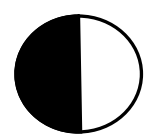

Moderadamente

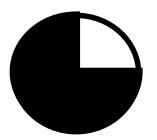

Bastante

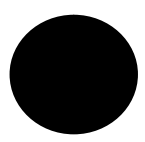

Totalmente

12. ¿En qué medida está satisfecho(a) con sus oportunidades de seguir logrando cosas en la vida?

13. ¿Cuánto siente que ma recibido el reconocimiento que merece en la vida?

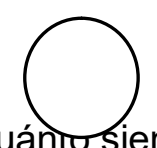

Nada

\begin{abstract}
Un poco
\end{abstract}
Moderadamente

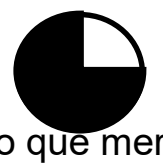

Bastante
Totalmente 


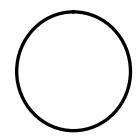

Nada

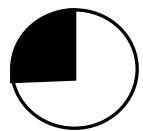

Un poco

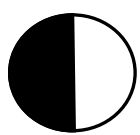

Moderadamente

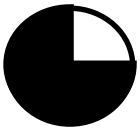

Bastante

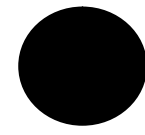

Totalmente

14. ¿En qué medida siente que tiene suficientes actividades a hacer cada día?

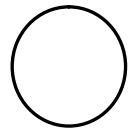

Nada

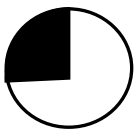

Un poco

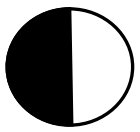

Moderadamente

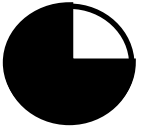

Bastante

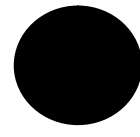

Totalmente

Las siguientes preguntas hacen referencia a que tan satisfecho(a), feliz o bien se ha sentido sobre varios aspectos de su vida en las dos últimas semanas, por ejemplo, sobre su participación en la vida comunitaria o sus logros en la vida:

15. ¿En qué grado está satisfecho(a) con lo que ha conseguido en la vida?

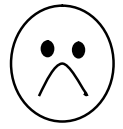

Muy insatisfecho

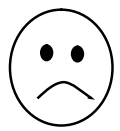

insatisfecho

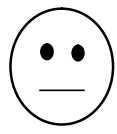

$\mathrm{Ni}$ insatisfecho(a) Ni satisfecho(a)

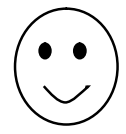

Satisfecho

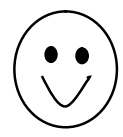

Muy satisfecho

16. ¿En qué grado está satisfecho(a) de la manera en que utiliza su tiempo?

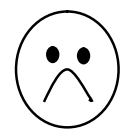

Muy insatisfecho

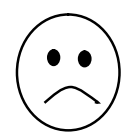

insatisfecho

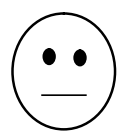

$\mathrm{Ni}$ insatisfecho(a) Ni satisfecho(a)

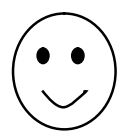

Satisfecho

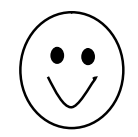

Muy satisfecho

17. ¿En qué grado está satisfecho(a) con su nivel de actividad?

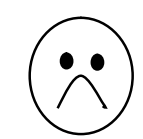

Muy insatisfecho

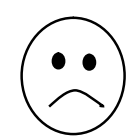

insatisfecho

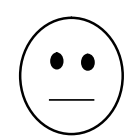

Ni insatisfecho(a) Ni satisfecho(a)

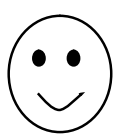

Satisfecho

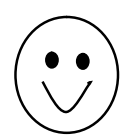

Muy satisfecho

18. ¿En qué grado está satisfecho(a) con sus oportunidades para participar en actividades de la comunidad?

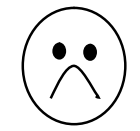

Muy insatisfecho

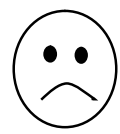

insatisfecho

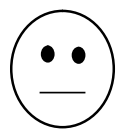

$\mathrm{Ni}$ insatisfecho(a) Ni satisfecho(a)

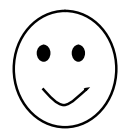

Satisfecho

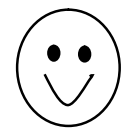

Muy satisfecho 
19. ¿En qué grado está contento(a) con las cosas que es capaz de tener ilusión?

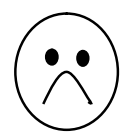

Muy descontento

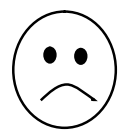

Descontento

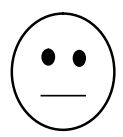

Ni descontento(a) $\mathrm{Ni}$ contento(a)

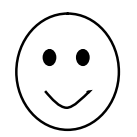

Contento

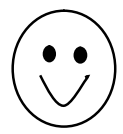

Muy contento

20. ¿Cómo calificaría el funcionamiento de su vista, oído, gusto, olfato y tacto?

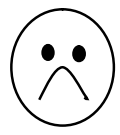

Muy mal

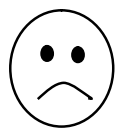

Mal

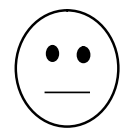

Ni mal ni bien

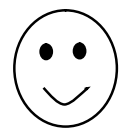

Bien

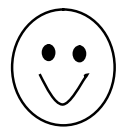

Muy bien

Las siguientes preguntas hacen referencia a cualquier relación íntima que pueda tener, por favor, considere estas preguntas con referencia a un compañero(a) u otra persona intima con quien usted comparte intimidad más que con cualquier otra persona en su vida:

21. ¿En qué medida tiene un sentimiento de compañía en su vida?

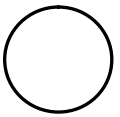

Nada

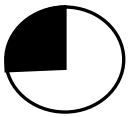

Un poco

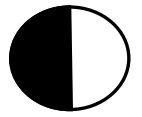

Lo normal

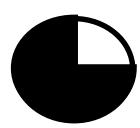

Bastante

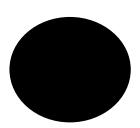

Extremadamente

22. ¿En qué medida experimenta amor en su vida?

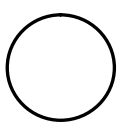

Nada

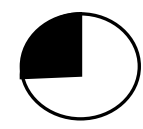

Un poco

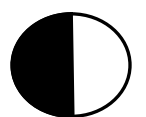

Lo normal

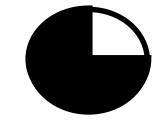

Bastante

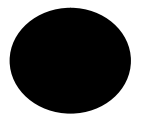

Extremadamente

23. ¿En qué medida tiene oportunidades para amar?

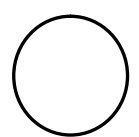

Nada

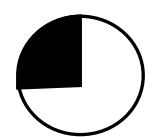

Un poco

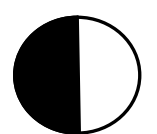

Moderadamente

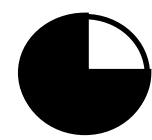

Bastante

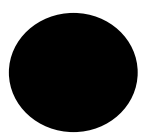

Totalmente

24. ¿En qué medida tiene oportunidades para ser amado?

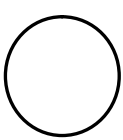

Nada

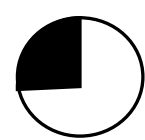

Un poco

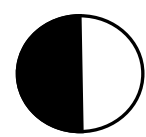

Moderadamente

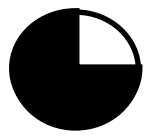

Bastante

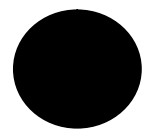

Totalmente 
